\title{
Preparation and functional characterization of human vascular endothelial growth factor-melittin fusion protein with analysis of the antitumor activity in vitro and in vivo
}

\author{
DINGDING WANG ${ }^{1,2}$, LILI HU ${ }^{1,2}$, MANMAN SU $^{3}, \mathrm{JU}_{\mathrm{WANG}^{4}}$ and TIANMIN XU $\mathrm{XU}^{5}$ \\ ${ }^{1}$ Department of Biotechnology, College of Life Science and Bio-pharmaceuticals; ${ }^{2}$ Guangdong Provincial Key Laboratory \\ of Biotechnology Candidate Drug Research, Guangdong Pharmaceutical University, Guangzhou, Guangdong; \\ ${ }^{3}$ Department of Regenerative Medicine, College of Pharmacy, Jilin University, Changchun, Jilin; ${ }^{4}$ Guangdong Provincial Key \\ Laboratory of Bio-engineering Medicine (National Engineering Research Centre of Genetic Medicine), Guangzhou, Guangdong; \\ ${ }^{5}$ Department of Obstetrics and Gynecology, The Second Clinical Hospital, Jilin University, Changchun, Jilin, P.R. China
}

Received May 9, 2015; Accepted June 22, 2015

DOI: $10.3892 /$ ijo.2015.3078

\begin{abstract}
Vascular endothelial growth factor and its tyrosine kinase receptors have been identified as key mediators of the regulation of pathologic blood vessel growth and maintenance in the promotion of angiogenesis and tumor growth. Therefore, an alternative approach to destroying tumor endothelium would be to make this tissue particularly sensitive to VEGF-mediated drug delivery. To verify this hypothesis, we generated a protein containing $\mathrm{VEGF}_{165}$ fused to melittin. Melittin is a small linear peptide composed of 26 amino acid residues that can exert toxic or inhibitory effects on many types of tumor cells. This protein is a cytolytic peptide that attacks lipid membranes, leading to significant toxicity. In the present study, the Pichia pastoris expression system was used to express the fusion protein. Under optimal conditions, stable $V_{E G F} F_{165}$-melittin production was achieved using a series of purification steps. The activity of $\mathrm{VEGF}_{165}$-melittin fusion protein was compared with melittin for its ability to suppress the growth of tumor cell line in vitro. The fusion toxin selectively inhibited growth of human hepatocellular carcinoma HepG-2 cell line with high expression of VEGFR-2. We found that sensitivity of VEGFR-2 transfected 293 cells to VEGF ${ }_{165^{-}}$ melittin enhanced as the cellular VEGFR-2 density increased. In an in vivo initial experiment, the fusion protein inhibited tumor growth in xenografts assays. Furthermore, successful expression and characterization of the fusion protein demon-
\end{abstract}

Correspondence to: Dr Tianmin Xu, Department of Obstetrics and Gynecology, The Second Hospital of Jilin University, $218 \mathrm{Zi}$ Qiang Street, Changchun, Jilin 130041, P.R. China

E-mail: tianminxu@aliyun.com

Abbreviations: PBS, phosphate-buffered saline; OPD, ortho-phenylenediamine; DAB, 3,3'-diaminobenzidine; BSA, bovine serum albumin

Key words: expression, fusion protein, vascular endothelial growth factor, melittin, antitumor strated its efficacy for use as a novel treatment strategy for cancer.

\section{Introduction}

The growth and metastatic spread of malignant tumors cannot proceed without the development of a vascular supply. Vascular endothelial growth factor-A (VEGF-A) plays a key role in tumor angiogenesis (1-4). The significant amount of VEGFR expression in the tumor vasculature presents a unique opportunity for therapeutic intervention. VEGF and its receptor VEGFR-1/VEGFR-2 provide an alternative approach for destroying tumor endothelium through targeting in combination with agents that kill cells, making them targets for the delivery of potent toxins to tumor endothelial cells $(5,6)$. VEGF mRNA is alternatively spliced, leading to proteins that are $208,189,165$, or 121 amino acids in length (7). $V_{E G F}$ and $\mathrm{VEGF}_{121}$ are secreted as soluble factors; however, $\mathrm{VEGF}_{208}$ and $\mathrm{VEGF}_{189}$ are secreted while binding to the extracellular matrix (8). Compared with $\mathrm{VEGF}_{121}, \mathrm{VEGF}_{165}$ retains a heparin-binding domain, which induces binding to the cell surface receptor. Furthermore, VEGF $_{165}$ is the most abundantly expressed splice variant (9). In the present study, we chose melittin for fusion with VEGF. This fusion protein, denoted as VEGFR VI65 -melittin, was shown to potently inhibit hepatocellular carcinoma and pancreatic cancer in vivo and in vitro.

Melittin is the principal toxic component in venom from the European honey bee Apis mellifera. This protein is a cationic, hemolytic and small linear peptide composed of 26 amino acid residues. Notably, the $\mathrm{N}$-terminus is predominantly hydrophobic and the $\mathrm{C}$-terminus is hydrophilic. Melittin has various effects, including antibacterial, antiviral, and antiinflammatory effects, in various cell types (10). It has been reported that melittin can induce apoptosis, cell cycle arrest and growth-inhibition in different tumor cells (11-13). However, the significant toxicity of melittin is achieved through a highly non-specific cytolytic attack of lipid membranes (14). The principle of the melittin toxicity is its physical and chemical 
destruction of cellular membranes, leading to a profound increase in the cell permeability barrier and leakage of cell contents $(15,16)$, thereby precluding any meaningful therapeutic benefit. An alternative approach for achieving practical therapeutic applications would be designing a new paradigm for the targeted delivery of potent toxins to tumor cells. Moreover, it has been reported that melittin suppresses tumor growth by targeting VEGF $(17,18)$. Therefore, melittin as a fusion partner should work well with VEGF.

In the present study, we prepared a novel fusion protein, VEGF $_{165}$-melittin, in Pichia pastoris. We generated an effective method for producing the recombinant protein in large quantities with high purity. Our results demonstrate that $\mathrm{VEGF}_{165}$-melittin retains functional activities including cytotoxicity and growth inhibition in HepG-2 and MHCC97-H human hepatocellular carcinoma cells in vitro. Furthermore, the fusion toxin was able to inhibit tumor growth in vivo. This fusion protein has the potential to be used as a new paradigm for the targeted delivery of cell-penetrating toxins to kill cancer cells in vitro and in vivo.

\section{Materials and methods}

Reagents and materials. Pichia pastoris X-33, the pPICZ $\alpha \mathrm{C}$ vector, and Zeocin antibiotic were obtained from Invitrogen (Carlsbad, CA, USA). Restriction enzymes, T4 DNA ligase, DNA marker, and the pMD-18T vector were purchased from Takara (Dalian, China). The protein marker was purchased from Thermo Fermentas and New England Biolabs (Guangzhou, China). All primers were synthesized by Shanghai Sangon Biotechnology Corp. (Shanghai, China). Anti-VEGF ${ }_{165}$, anti-VEGFR-1, anti-VEGFR-2, anti-melittin, HRP-goat antirabbit conjugate and HRP-goat anti-mouse conjugate were obtained from Santa Cruz Biotechnology (Santa Cruz, CA, USA). Melittin was purchased from Nanning Innovation and Technology Pharmaceutical Co., Ltd. (Guangxi, China). Anti-VEGF blocking antibody was purchased from R\&D Systems (Minneapolis, MN, USA). VEGFR-2/KDR gene was purchased from Sino Biological Inc. (Beijing, China).

Human hepatocellular carcinoma cell lines (HepG-2 and MHCC97-H), a human pancreatic adenocarcinoma cell line (AsPC-1), and 293 human primary embryonic kidney cells were obtained from the American Type Culture Collection. All the cells were passaged according to their protocol from ATCC, and no more than 6 months elapsed after the resuscitation and culturing of the cells. Serum and culture medium were purchased from Invitrogen. BALB/c mice and BALB/c nude mice (4-5 weeks) were obtained from the Experimental Animal Research Centre of Zhongshan University and raised in its laboratory. All animal protocols followed the National Guidelines for the Care and Use of Animals.

Yeast culture media. Pichia pastoris was cultured in YPD medium containing $10 \mathrm{~g} / \mathrm{l}$ yeast extract, $20 \mathrm{~g} / \mathrm{l}$ peptone and $20 \mathrm{~g} / \mathrm{l} \mathrm{D}$-glucose. To prepare YPD plates, 2\% agar (w/v) was added into YPD medium. YPD-Zeocin plates containing $0.1 \mathrm{mg} / \mathrm{ml}$ Zeocin were used for the selection of transformants. The Pichia pastoris cells were grown in BMGY medium (1\% yeast extract, $2 \%$ peptone, $1 \%$ glycerol, $1.34 \%$ yeast nitrogen base and $0.1 \mathrm{M}$ potassium phosphate, $\mathrm{pH}$ 6.0) and BMMY medium (1\% yeast extract, $2 \%$ peptone, $0.5 \%$ methanol, $1.34 \%$ yeast nitrogen base and $0.1 \mathrm{M}$ potassium phosphate, $\mathrm{pH}$ 6.0) for induction.

Construction of expression vector containing pPICZ $\alpha \mathrm{C} /$ $V E G F_{165}$-melittin. A DNA insert encoding melittin was prepared via artificial synthesis. A linker containing $(\mathrm{GGGGS})_{4}$, EcoRI, ApaI, AccI and $\mathrm{XbaI}$ sequences were appended when the synthetic fragment was designed. Then, the melittin DNA fragment was digested with EcoRI and $\mathrm{XbaI}$ and ligated into a linearized $\mathrm{pPICZ} \alpha \mathrm{C}$ vector to generate the plasmid pPICZ $\alpha \mathrm{C} /$ melittin.

To clone the $\mathrm{VEGF}_{165}$ gene, reverse-transcription polymerase chain reaction (RT-PCR) was performed with the primers 5'-ATT CTC GAG AAG AGA GCA CCC ATG GCA GAA GGA G-3' (forward) and 5'-GTA GAA TTC CCG CCT CGG CTT GTC ACA TTT TTA-3' (reverse), and total RNA extracted from human hepatoma (HepG2) cells served as the template. Following digestion with $X h o I$ and EcoRI, the PCR fragment was cloned into $\mathrm{pPICZ} \alpha \mathrm{C} /$ melittin and treated with the same endonucleases to generate the recombinant eukaryotic expression plasmid pPICZ $\alpha \mathrm{C} / \mathrm{VEGF}_{165}$-melittin. The recombinant plasmid was confirmed by restriction analysis and sequencing.

Transformation and screening of recombinant strains. Recombinant plasmid DNA was linearized with SacI and then transformed into Pichia pastoris X-33 by electroporation using a MicroPulser (Bio-Rad Laboratories, Hercules, CA, USA) following the pPICZ $\alpha \mathrm{C}$ vector manual. The yeast strains transformed with empty vector $\mathrm{pPICZ} \alpha \mathrm{C}$ plasmid served as a negative control. The cells were spread on YPD plates containing Zeocin at 100, 250, 500 and $1,000 \mathrm{mg} / \mathrm{ml}$ and incubated at $28^{\circ} \mathrm{C}$. Colonies appeared after 2-3 days of incubation at $28^{\circ} \mathrm{C}$. The inserted foreign gene in the genomic DNA of transformants were detected by PCR assay using the primers mentioned above. Thirty cycles of PCR were performed with incubations for $30 \mathrm{sec}$ at $94^{\circ} \mathrm{C}, 30 \mathrm{sec}$ at $55^{\circ} \mathrm{C}$ and $1.5 \mathrm{~min}$ at $72^{\circ} \mathrm{C}$.

Optimized expression of the fusion protein in P. pastoris. To confirm the optimal expression conditions for the fusion protein, various culture parameters, including induction time-points and $\mathrm{pH}$ values ( $\mathrm{pH} 3.0-7.0$ with $0.5 \mathrm{pH}$ intervals), were evaluated. The processes were the same as above. At specific intervals, $0.5 \mathrm{ml}$ cell suspensions were removed and then substituted with the same volume of fresh medium. The cell culture supernatant was tested by ELISA assays.

Purification of VEGF 165 -melittin. $\mathrm{VEGF}_{165}$-melittin production was scaled up in 21 BMGY medium based on the process introduced in the Invitrogen manual (19). Transformants were cultured at $28^{\circ} \mathrm{C}\left(\mathrm{pH}\right.$ 6.0) until the culture reached $\mathrm{OD}_{600}=2.0$ 6.0 , the cells were harvested by centrifugation, redissolved in $21 \mathrm{BMMY}$ medium, and cultured at $28^{\circ} \mathrm{C}$ with oscillation for $72 \mathrm{~h}$. The fermentation broth was supplemented every $24 \mathrm{~h}$ with $10 \mathrm{ml}$ methanol to maintain the induced control.

Fermentation supernatant was collected by filtration $(0.45 \mu \mathrm{m})$ after harvesting by centrifugation at $12,000 \mathrm{r} / \mathrm{min}$ for 15 min. A Ni ${ }^{2+}$ NTA column (GE Healthcare, Piscataway, 
NJ, USA) was equilibrated in binding buffer $(20 \mathrm{mM}$ $\mathrm{Na}_{3} \mathrm{PO}_{4} \cdot 12 \mathrm{H}_{2} \mathrm{O}, \mathrm{pH}$ 7.4, $0.5 \mathrm{M} \mathrm{NaCl}$ and $30 \mathrm{mM}$ imidazole). The supernatant was diluted 3 -fold with binding buffer and loaded onto a $\mathrm{Ni}^{2+}$ NTA column at a speed of $0.5 \mathrm{ml} / \mathrm{min}$. Then, the column was washed with the same buffer at a rate of $1.0 \mathrm{ml} / \mathrm{min}$ to eliminate unbound proteins. Bound protein was then eluted from the column with $20 \mathrm{mM} \mathrm{Na}_{3} \mathrm{PO}_{4} \cdot 12 \mathrm{H}_{2} \mathrm{O}$, $\mathrm{pH} 7.4,0.5 \mathrm{M} \mathrm{NaCl}$ and $0.2 \mathrm{M}$ imidazole at a rate of $0.8 \mathrm{ml} / \mathrm{min}$. Eluted protein was then transferred to storage buffer (1X PBS) by chromatography using a Thermo Scientific Zeba desalting column (Thermo Fisher Scientific, Waltham, MA USA).

Protein assay. The protein concentrations of the samples were measured using the Bradford assay with bovine serum albumin as a standard.

Enzyme-linked immunosorbent assay. Individual wells of ELISA plates (Costar) were coated with fusion toxin sample supernatants and coating buffer $\left(\mathrm{Na}_{2} \mathrm{CO}_{3}-\mathrm{NaHCO}_{3}, \mathrm{pH} 9.6\right.$, dilution: $2 \mu \mathrm{g} / 100 \mu \mathrm{l}$ ) overnight at $4^{\circ} \mathrm{C}$. The plates were blocked with $2 \%$ BSA in TPBS (PBS ${ }^{1}, 0.1 \%$ Tween-20, pH 7.2) and incubated for $2 \mathrm{~h}$ at room temperature. The primary antibody against rabbit was used at 1:1,000 and precoated for $2 \mathrm{~h}$ at $37^{\circ} \mathrm{C}$. After several washes with TPBS, the plates were incubated with goat anti-rabbit IgG conjugated to $\operatorname{HRP}(1: 2,000$ dilutions at blocking buffer) for $2 \mathrm{~h}$. The color reaction was implemented with OPD zymolyte containing $0.02 \% \mathrm{H}_{2} \mathrm{O}_{2}$, and the plates were incubated for $15 \mathrm{~min}$ at room temperature in the dark. Then, $50 \mu \mathrm{l}$ of $\mathrm{H}_{2} \mathrm{SO}_{4}$ solution $(2 \mathrm{M})$ was used to stop the reaction. Absorbance values at $490 \mathrm{~nm}$ were read using an ELX800 microplate reader (Bio-Tek Instruments Inc., Winooski, VT, USA). After adding stop solution, plate reads were completed within $2 \mathrm{~h}$.

SDS-PAGE and western blot assays. Cell lysates were separated by SDS-PAGE in $10 \%$ gels and transferred to a polyvinylidene difluoride (PVDF) membrane (Millipore) using a semi-dry electroblotting apparatus (Bio-Rad Laboratories) at $200 \mathrm{~mA}$ for $1 \mathrm{~h}$ in Towbin transfer buffer $(25 \mathrm{mM}$ Tris and $192 \mathrm{~mm}$ glycine). The membrane was blocked with $2 \%$ BSA for $1.5 \mathrm{~h}$ at room temperature. Then, the membrane was incubated with primary antibodies against rabbit for $12 \mathrm{~h}$. After washing, the membrane was incubated with a goat anti-rabbit IgG antibody conjugated to HRP (Weijia, Shaanxi, China) that was diluted 1:250. The bound antibody was developed with 3,30-diaminobenzidine (DAB).

$N$-terminal amino acid sequence and mass spectrometric analyses. The N-terminal amino acid sequence of the $\mathrm{VEGF}_{165}$ melittin fusion protein was determined by automated Edman degradation, which was performed with a model protein sequencer-491 (Applied Biosystems, Foster City, CA, USA). The purified protein was adsorbed onto a PVDF membrane (ProSorb) and sequenced using established protocols. Mass spectrometric analysis of $\mathrm{VEGF}_{165}$-melittin was performed with an autoflex speed MALDI-TOF/TOF MS (Brucker Daltonics, Billerica, MA, USA).

Reverse transcription-polymerase chain reaction. Total cellular RNA was extracted from cell cultures using the
RNAiso reagent (Takara, Tokyo, Japan) according to the manufacturer's protocol. RNA concentration was detected using a BioPhotometer (Eppendorf Scientific, Hamburg, Germany). Reverse transcription of total RNA primed with an oligo(dT) oligonucleotide was done with M-MLV reverse transcriptase (Promega, Mannheim, Germany) according to the instructions of the manufacturer. First-strand complementary DNA was amplified using Takara Ex Taq (Takara).

The primers for the respective genes were designed as follows: VEGF, 5'-GCA CCC ATG GCA GAA GGA-3' (forward) and 5'-TTC TGT ATC AGT CTT TCC-3' (reverse); VEGFR-1, 5'-GAA GGC ATG AGG ATG AGA-3' (forward) and 5'-CAG GCT CAT GAA CTT GAA-3' (reverse); KDR/VEGFR-2, 5'-CAT GTA CGG TCT ATG CCA-3' (forward) and 5'-CGT TGG CGC ACT CTT CCT-3' (reverse); and $\beta$-actin, 5'-TTC CTG GGC ATG GAG TCC-3' (forward) and 5'-CGC CTA GAA GCA TTT GCG-3' (reverse). RT-PCR products were analyzed by electrophoresis on a $1 \%$ agarose gel.

Cytotoxicity assay. Cells were seeded in 96-well plates at $5-10 \times 10^{4}$ cells/well. Cells were then starved with phenol red-free Dulbecco's modified Eagle's medium plus 1\% dialyzed fetal calf serum (A15-107; PAA Laboratories, Dartmouth, MA, USA) for $24 \mathrm{~h}$. The experiment included six $\mathrm{VEGF}_{165}$-melittin fusion protein groups $(0,0.8,1.6,3.2,6.4$ and $12.8 \mu \mathrm{g} / \mathrm{ml})$. Human primary embryonic kidney cells $(n=293)$ were used for control. Cell growth was induced by the fusion toxin for $48 \mathrm{~h}$ and then measured with the MTT assay. Absorbance at $570 \mathrm{~nm}$ was detected with a reference at $630 \mathrm{~nm}$ serving as a blank. The influence of the fusion toxin on cell activity was evaluated and compared with control. The control cells were set to $100 \%$ activity. The mean value of 5 wells was counted, and triplicates were used in each experiment.

To test VEGFR-mediated effects of VEGF $_{165}$-melittin fusion protein on the proliferation and viability of human cancer cells, HepG-2 cells was used for subsequent studies. HepG-2 cells were cultured as described above. Five experimental groups were designed and HepG-2 untreated was the control group.

Inhibitory effects of VEGF ${ }_{165}$-melittin on hepatocellular carcinoma and pancreatic cancer xenografts in nude mice. After hypodermic injection of $2.5 \times 10^{7} \mathrm{HepG}-2$ or $5 \times 10^{6}$ AsPC-1 cells in BALB/c athymic nude mice, initial tumors were observed on day 21. Afterward, all mice in the experimental groups were intravenously injected with $0.2 \mathrm{mg}$ VEGF $_{165}$-melittin daily for 28 days, and PBS was used as a control. The subcutaneous tumor parameters were measured every day, including the length, width and height. The tumor volume $\left(\mathrm{mm}^{3}\right)$ was estimated according to the equation $\mathrm{a}^{2} \mathrm{~b} / 2$, where $\mathrm{a}$ is the short diameter $(\mathrm{mm})$ and $\mathrm{b}$ is the long diameter $(\mathrm{mm})$. The tumor weights were measured after the mice were sacrificed. The tumor samples were maintained in formalin, and an assessment of mortality was performed.

\section{Results}

$V E G F_{165}$-melittin expression and optimization. A plasmid was created to express the $\mathrm{VEGF}_{165}$ fragment fused to melittin to 

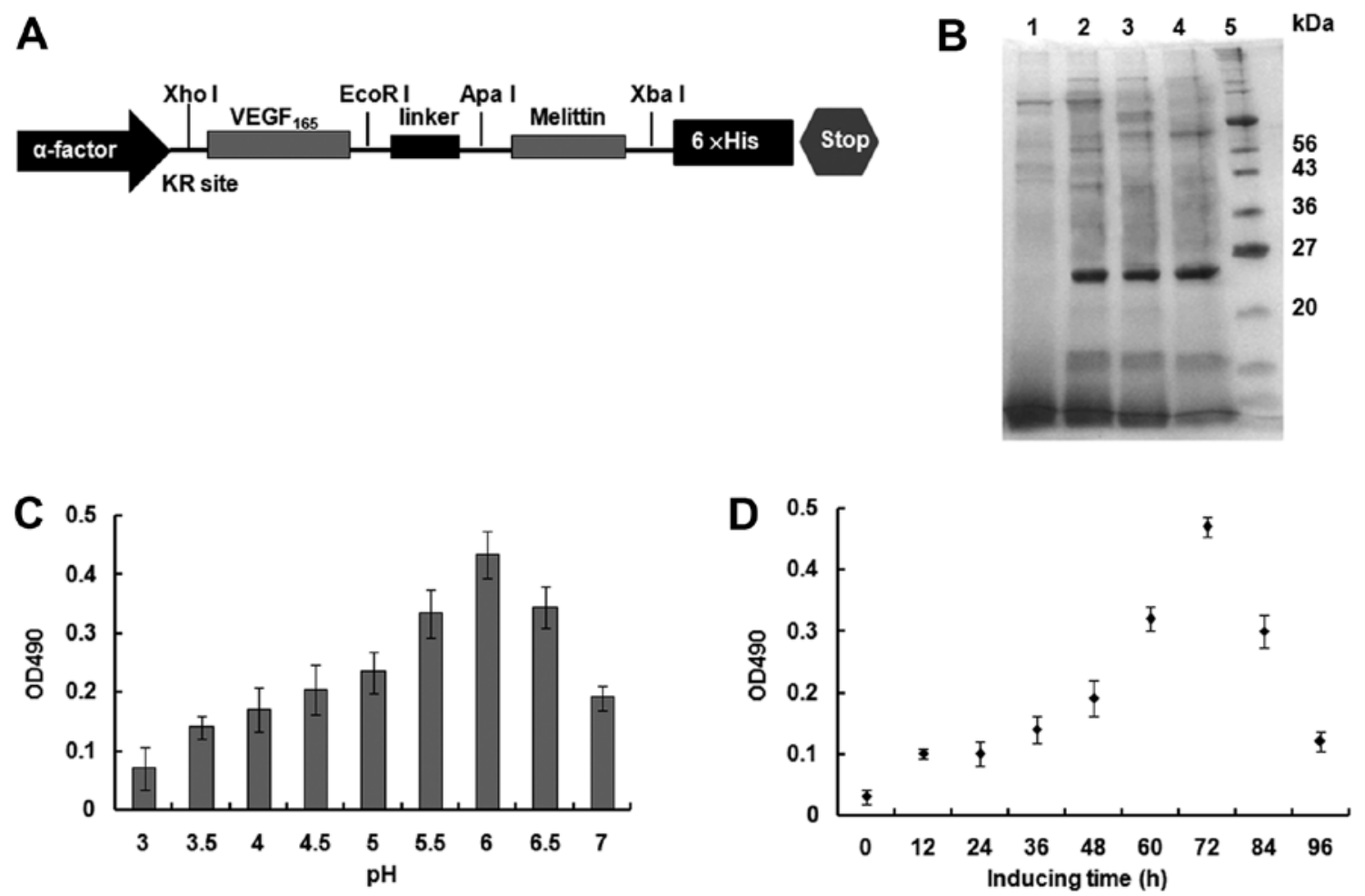

Figure 1. (A) Construction and details of the cloning sites of $\mathrm{VEGF}_{165}$-melittin (B) SDS-PAGE analysis of fusion protein. Lane 1, supernatant of transformant with pPICZ $\alpha \mathrm{C}$ empty plasmid grown under identical condition. Lanes 2, 3 and 4, samples from different transformants; lane 5, protein molecular weight marker. (C and D) Optimization of $\mathrm{VEGF}_{165}$-melittin in the $50 \mathrm{ml}$ conical tube (the $\mathrm{pH}$ value and induction time-points). Supernatants collected at each evaluated condition were processed by ELISA. Optimization of the $\mathrm{pH}$ value. The experiments were performed three times, and the mean values $\pm \mathrm{SD}$ are presented.

Table I. Summary of purification process of $\mathrm{VEGF}_{165}$-melittin from 2 liters of culture supernatant purification.

\begin{tabular}{lcccc}
\hline Purification steps & Total protein $(\mathrm{mg} / \mathrm{l})$ & $\mathrm{VEGF}_{165}$-melittin $(\mathrm{mg} / \mathrm{l})$ & Purity $(\%)$ & Recovery $(\%)$ \\
\hline Supernatants & 256.5 & 154.2 & 60.1 & \\
$\mathrm{Ni}^{2+}$-NTA & 121.1 & 115.3 & 95.2 & 74.8 \\
Desalting column & 84.3 & 80.3 & 95.3 & 52.1 \\
\hline
\end{tabular}

generate a $25 \mathrm{kD} \mathrm{VEGF}_{165}$-melittin fusion toxin. The structure of the details of $\mathrm{VEGF}_{165}$-melittin is shown in Fig. 1A. $\mathrm{pPICZ} \alpha \mathrm{C} /$ melittin is based on the Pichia pastoris expression vector pPICZ $\alpha \mathrm{C}$. This vector was used to express the $\mathrm{VEGF}_{165^{-}}$ melittin fusion protein, which is composed of the melittin fragment cut from the $A p a \mathrm{I}$ and $X b a \mathrm{I}$ sites following the VEGF $_{165}$ fragment. A linker, (GGGGS), was synthesized for spatial configuration of the fusion toxin. The $\mathrm{VEGF}_{165}$ sequence was amplified and inserted into the pPICZ $\alpha \mathrm{C} /$ melittin expression vector to create $\mathrm{pPICZ} \alpha \mathrm{C} / \mathrm{VEGF}_{165}$-melittin. Sequence analysis of the plasmid DNA was used to confirm integration in positive colonies.

After electroporation with SacI-linearized pPICZaC/ VEGF $_{165}$-melittin, $90 \%$ of transformants were Mut ${ }^{+}$. PCR analysis of genomic DNA demonstrated that the gene of interest was integrated into the stable transformants, and no similar bands were observed for negative control samples.

The positive transformants were germinated in BMGY medium and induced in BMMY medium at $28^{\circ} \mathrm{C}$ for 7 days. The volume of the culture medium was $10 \mathrm{ml}$. After 3 days, the culture supernatants were analyzed by SDS-PAGE. The results indicated that the molecular weight of $\mathrm{VEGF}_{165}$-melittin was consistent with the predicted size of $25 \mathrm{kDa}$ (Fig. 1B).

Transformants expressing a high level of fusion protein were selected, and one was chosen for the scaling up. Based on analysis of optimized expression conditions, the parameters used were as follows: $\mathrm{pH}$ : 6.0, induction time-point: $72 \mathrm{~h}$, and final methanol concentration: $0.5 \%$ (v/v) (Fig. 1C and D).

$V E G F_{165}$-melitin fermentation and purification. $V G_{165}$-melittin supernatant was purified by $\mathrm{Ni}^{2+}$ affinity chromatography and Thermo Scientific Zeba desalting column chromatography. Following these processes, $\sim 160 \mathrm{mg}$ pure recombinant protein was obtained from 21 fermentation liquor. SDS-PAGE analysis demonstrated that the purity of VEGF $_{165}$-melittin was $~ 95 \%$ (Fig. 2A). At every step of purification, the recovery, purity and yield of the fusion toxin were estimated as shown in Table I.

Western blot assays were used to preliminarily evaluate the purified recombinant protein. The identity of $\mathrm{VEGF}_{165}$-melittin 


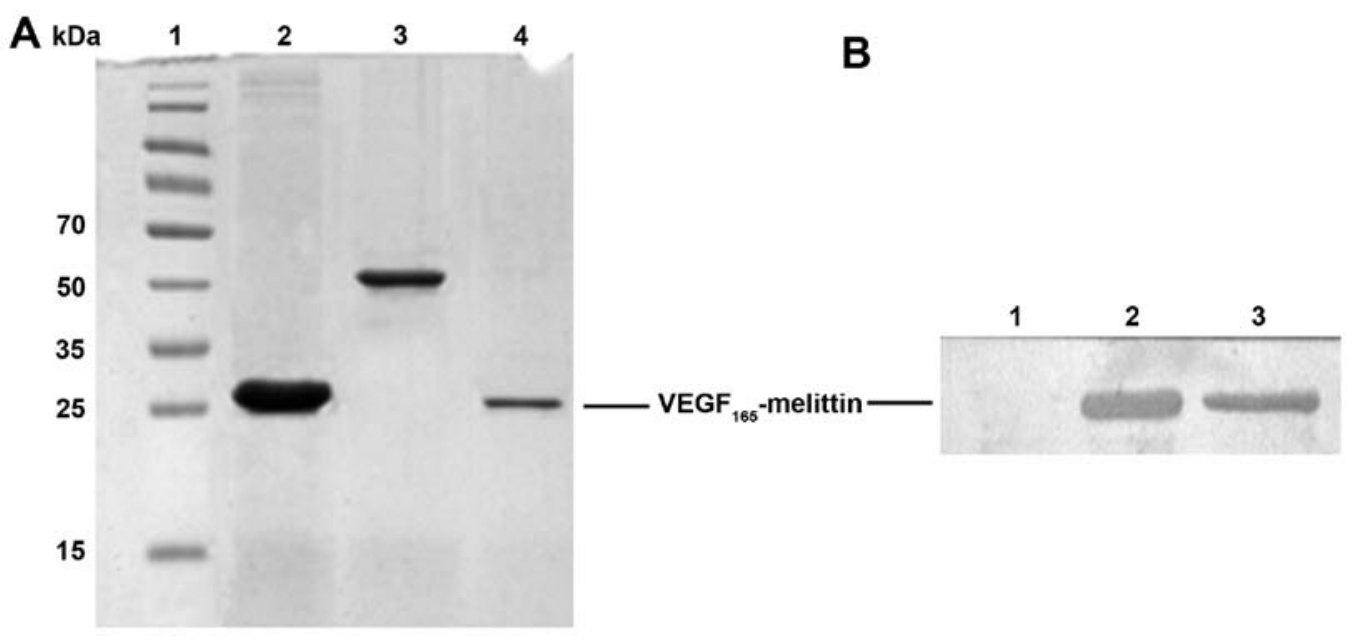

Figure 2. (A) SDS-PAGE analysis of purified $\mathrm{VEGF}_{165}$-melittin fusion protein. Lane 1, protein molecular weight marker. Lane 2, $50 \mu 1$ samples from purified supernatant (reduced). Lane 3, $30 \mu \mathrm{l}$ samples from purified supernatant (non-reduced). Lane 4, $20 \mu 1$ samples from purified supernatant (reduced). (B) Western blot analysis of $\mathrm{VEGF}_{165}$-melittin. Lane 1, supernatant of transformant with the plasmid pPICZ $\alpha \mathrm{C}$ as a negative control. Lanes 2 and 3 , samples from purified supernatant.

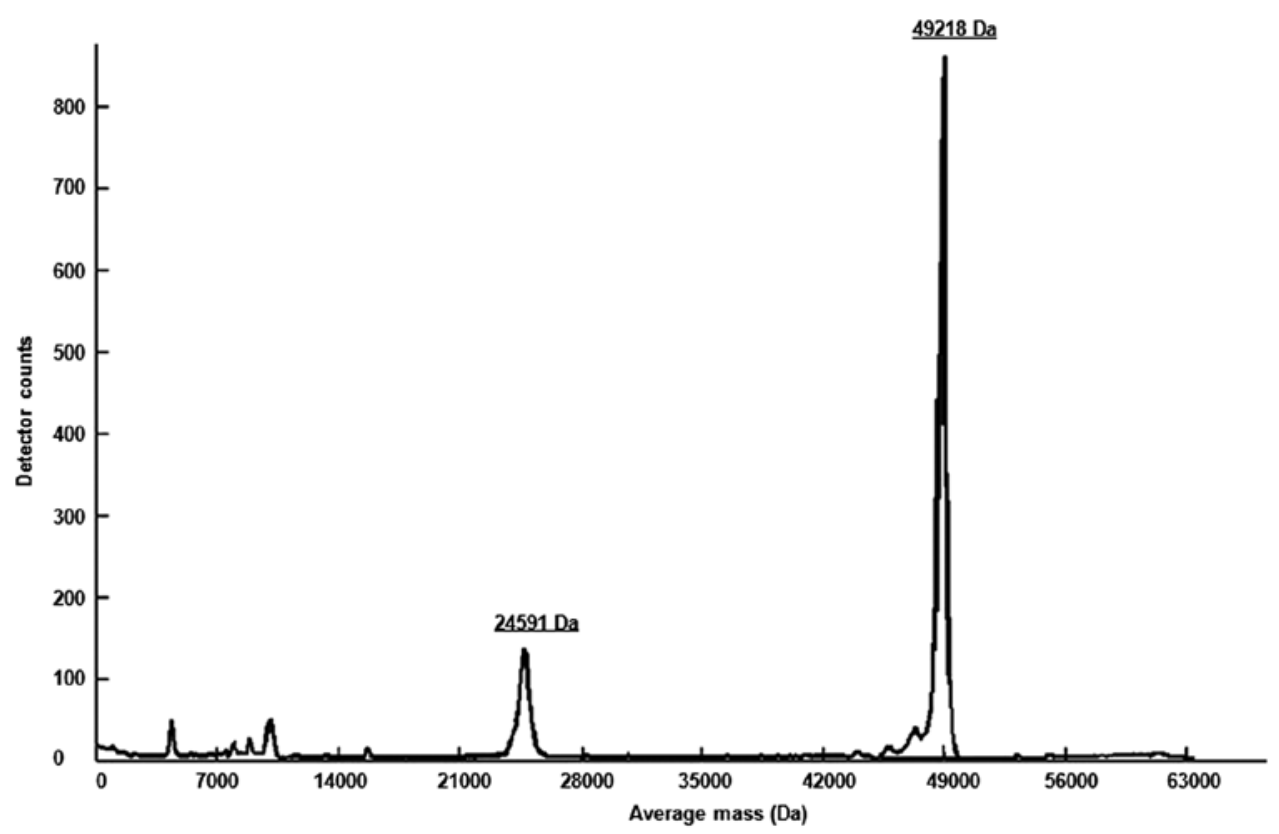

Figure 3. Mass spectrometry analysis of $\mathrm{VEGF}_{165}$-melittin. $\mathrm{VEGF}_{165}$-melittin showed two major peaks in 49218 Da as dimer and 24591 Da as monomer. Observed average masses resulting from the proteins are reported in Da.

was confirmed by immunoreactivity with a rabbit anti-human VEGF $_{165}$ polyclonal antibody (Fig. 2B). The results were consistent with our expectations. No band was observed in lane 1 , which contains the supernatant of the X33 pPICZ $\alpha \mathrm{C}$ transformant.

Molecular weight and N-terminal sequencing analyses. To verify the molecular weight and integrity of the recombinant protein, mass spectrometry was performed using purified $\mathrm{VEGF}_{165}$-melittin. The expected molecular mass $\mathrm{VEGF}_{165^{-}}$ melittin is 221 amino acids, and it primarily exists in solution as a homodimer due to a disulfide linkage in the linker. The results of the molecular weight analysis of the fusion toxin are shown in Fig. 3, and they are in accordance with our previous results, indicating that the purified recombinant toxin is the expected $\mathrm{VEGF}_{165}$-melittin protein.

According to $\mathrm{N}$-terminal sequencing analysis, the first 15 amino acids of the purified peptide were A P M A E G G G Q N H H E V V. These were consistent with the N-terminal sequence of $\mathrm{VEGF}_{165}$-melittin, thus indicating successful expression and purification of this protein.

Cytotoxicity assay. The effects of $\mathrm{VEGF}_{165}$-melittin on the proliferation and viability of human hepatocellular carcinoma cell lines (HepG-2 and MHCC97-H), human pancreatic adenocarcinoma cell lines AsPC-1 and human primary embryonic kidney cells 293 were studied for a 72-h period. The fusion protein was applied to the cells at seven 


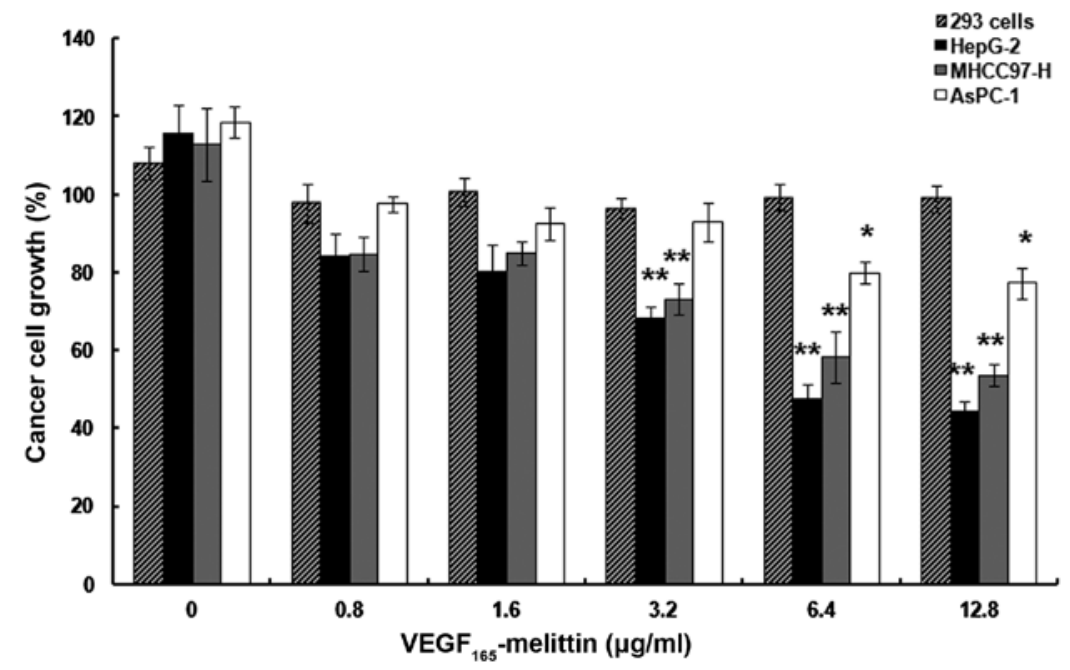

Figure 4. MTT assays to evaluate the inhibition effect to the proliferation of cancer cell lines. Each bar represents the cell growth of HepG-2 treated with fusion toxin (dark, ${ }^{* *} \mathrm{P}<0.01$ ), MHCC97-H cells (gray, ${ }^{* * *} \mathrm{P}<0.01$ ), AsPC-1 cells (white bar, ${ }^{*} \mathrm{P}<0.05$ ) and 293 cells (stripes). The experiments were performed three times, and the mean values $\pm \mathrm{SD}$ are presented.

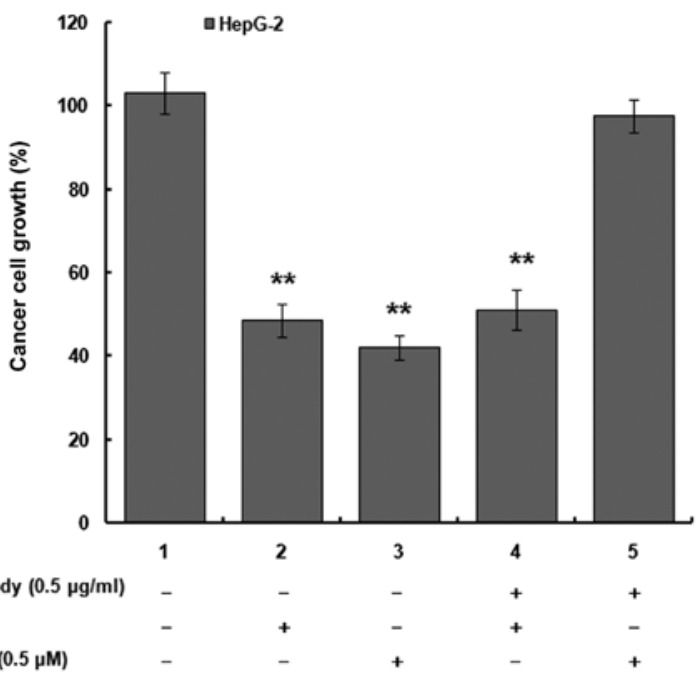

Figure 5 . The cytotoxicity of $\mathrm{VEGF}_{165}$-melittin was inhibited by anti-VEGF blocking antibody $\left({ }^{* *} \mathrm{P}<0.01\right)$. The experiments were performed three times, and the mean values $\pm \mathrm{SD}$ are presented.

different final concentrations. Fig. 4 shows the proliferation and viability changes that occurred during treatment. Cell counts and an MTT-assay indicated that the fusion toxin influenced the proliferation of HepG-2 and MHCC97-H cells more significantly than that of AsPC-1 cells. The proliferation of the HepG-2 cells significantly decreased by $55 \%$ in an MTT assay $(\mathrm{P}<0.01)$. However, an effect of the fusion toxin on the viability of 293 cells was not observed, even at the highest $\mathrm{VEGF}_{165}$-melittin dose.

To further assess the mediation effects between $\mathrm{VEGF}_{165^{-}}$ melittin and VEGFR inhibition, human hepatocellular carcinoma cells HepG-2 were incubated with $\mathrm{VEGF}_{165^{-}}$ melittin or melittin for $48 \mathrm{~h}$ in the presence or absence of $0.5 \mu \mathrm{g} / \mathrm{ml}$ anti-VEGF antibody. Fig. 5 shows the proliferation of the HepG-2 cells significantly decreased when melittin or VEGF $_{165}$-melittin was added $(\mathrm{P}<0.01)$. However, in $\mathrm{VEGF}_{165^{-}}$ melittin groups, the inhibitory activity was not observed after
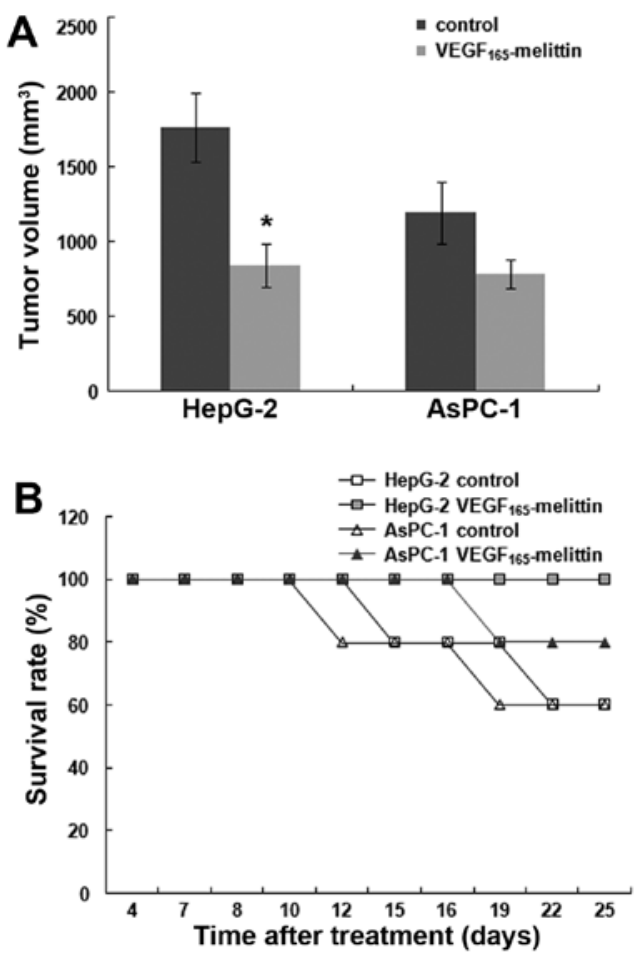

Figure 6. Inhibition of HepG-2 and AsPC-1 xenografts in nude mice by VEGF $_{165}$-melittin. (A) Tumor volume of the $\mathrm{VEGF}_{165}$-melittin group and the control group ( ${ }^{*} \mathrm{P}<0.05$ ). (B) Survival analysis of the $\mathrm{VEGF}_{165}$-melittin group and the control group.

incubated with the anti-VEGF antibody. This sensitivity of HepG-2 might be mediated by VEGFR present on HepG-2 cells, since 293 cells without known VEGF receptors were not affected by $\mathrm{VEGF}_{165}$-melittin at high concentrations (Fig. 4). Presence of VEGFR appears to be necessary for induction of HepG-2 cell death by $\mathrm{VEGF}_{165}$-melittin.

$V E G F_{165}$-melittin-mediated tumor growth inhibition in vivo. In the HepG-2 xenograft nude mouse model, the average tumor volume in $\mathrm{VEGF}_{165}$-melittin mice was $843 \mathrm{~mm}^{3}$, and 

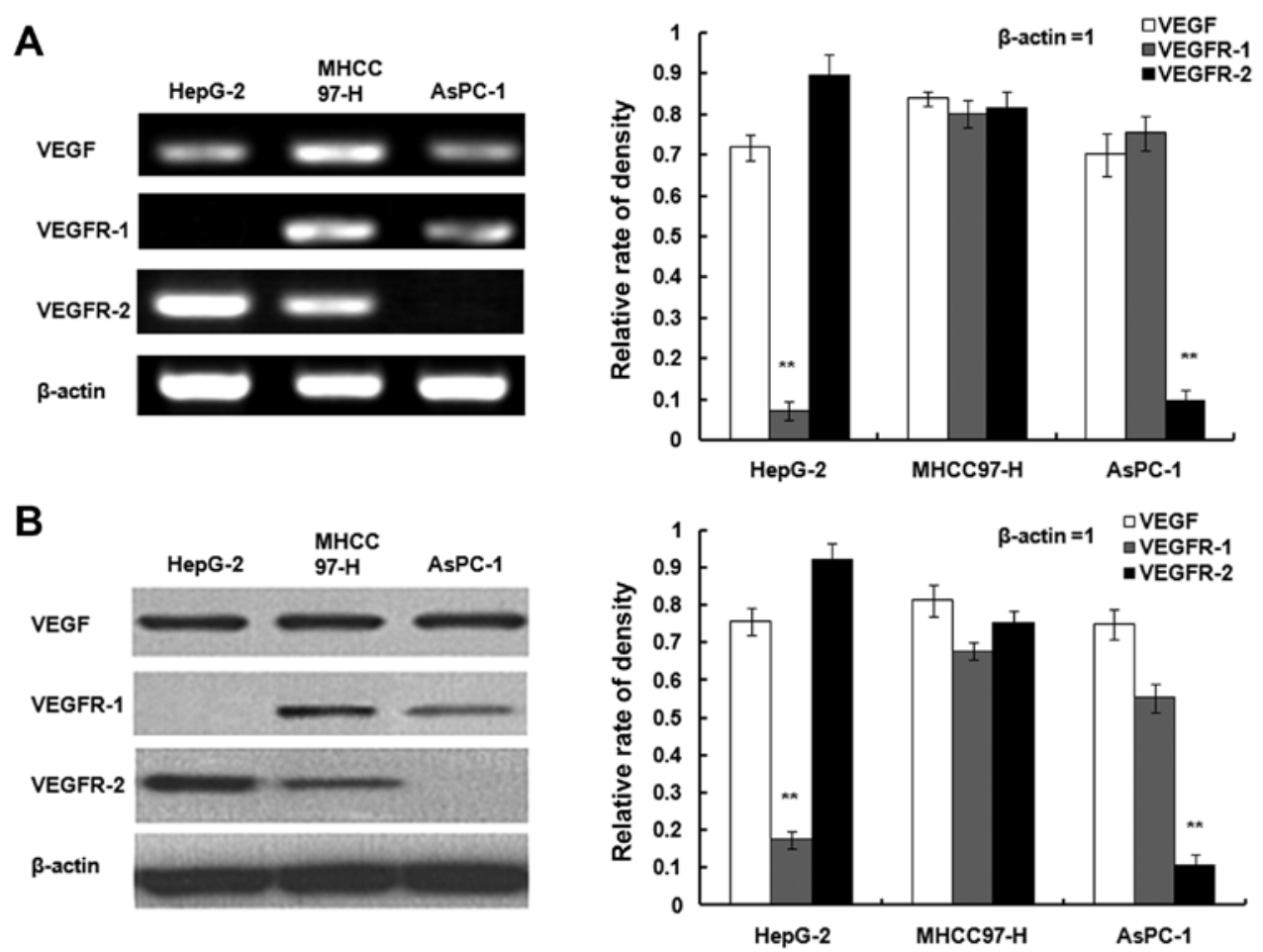

Figure 7. (A) RT-PCR analysis of expression of VEGF, VEGFR-1 and KDR/VEGFR-2 in HepG-2, MHCC97-H and AsPC-1 cells. (B) Western blot analysis $\left({ }^{* *} \mathrm{P}<0.01\right)$. The data represent the mean values $\pm \mathrm{SD}$ of two experiments, each in duplicate.
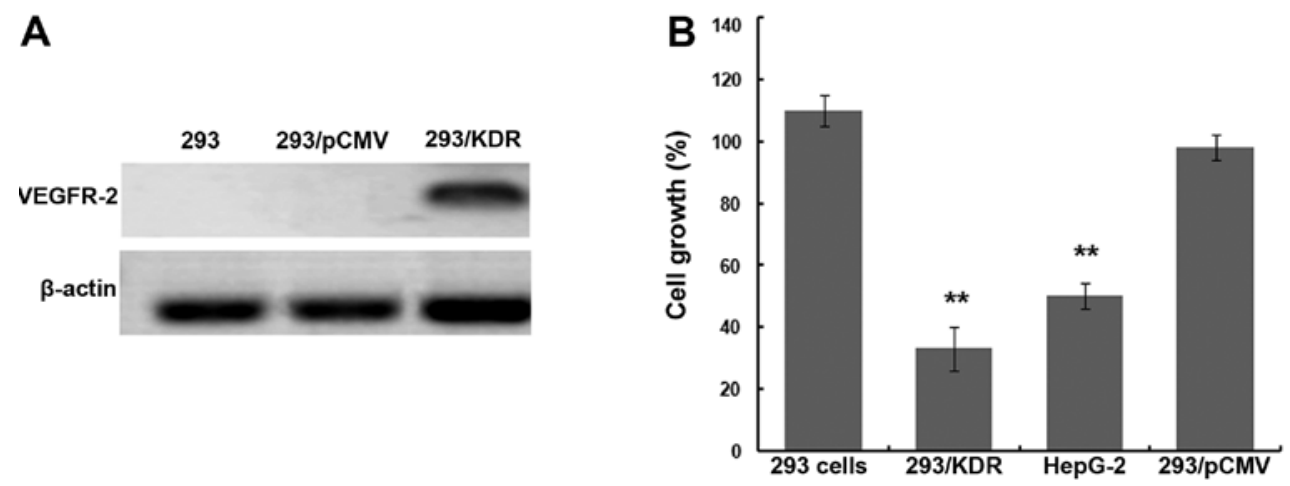

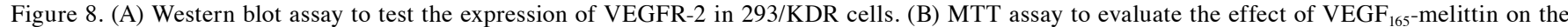
proliferation and viability of $293,293 / \mathrm{KDR}$, HepG-2 and $293 / \mathrm{pCMV}$ cells $\left({ }^{* * *} \mathrm{P}<0.01\right)$. The experiments were performed three times, and the mean values \pm SD are presented.

it was $1,769 \mathrm{~mm}^{3}$ in control mice (Fig. 6A). Therefore, the inhibitory rate of the average tumor volume was $52.3 \%$. Twenty-eight days after treatment with the fusion toxin, the survival was $100 \%$ for $\mathrm{VEGF}_{165}$-melittin mice and $60 \%$ for control mice (Fig. 6B). In the AsPC-1 xenograft nude mouse model, inhibition of the average tumor volume in the experimental group was $34.4 \%$ as compared with the control group. Significant $\mathrm{VEGF}_{165}$-melittin-mediated inhibition of tumor growth was demonstrated. Based on these results, it was obvious that there were stronger effects in HepG-2 compared with AsPC-1 cells, which suggests that the high expression level of VEGFR-2 in HepG-2 might mediate this influence.

Specific toxicity of VEGF ${ }_{165}$-melittin targeting VEGFR-2. Expression of VEGF, VEGFR-1 and KDR/VEGFR-2 in
HepG-2 and MHCC97-H cells as well as AsPC-1 cells were determined with RT-PCR and western blot assays (Fig. 7A and B). All three cell lines exhibited VEGF. MHCC-97H cells were positive for VEGFR-1 and KDR/VEGFR-2. KDR/VEGFR-2 was expressed in HepG-2 and VEGFR1 was expressed in AsPC-1.

Furthermore, KDR/VEGFR-2 was overexpressed in 293 cells to evaluate the specific targeting of fusion protein (Fig. 8A). The effect of $\mathrm{VEGF}_{165}$-melittin on the proliferation and viability of 293 cells, 293 transfected with pCMVp-NEOBAN/KDR plasmid (293/KDR) and HepG-2 cells was studied. The 293 cells transfected with pCMVp-NEO-BAN empty plasmid (293/pCMV) were used as control. In targeted cells, the cytotoxicity of $\mathrm{VEGF}_{165}$-melittin was strongly dependent on the VEGFR-2 density. Fig. 8B shows proliferation and viability changes using the MTT assay. 
Statistical analysis. Statistical analysis was performed using Statistical Package for Social Sciences (SPSS) 13.0 software. Data are presented as the means \pm SD. Statistical significance was determined by one-way analysis of variance or the t-test. P-values $<0.05$ were considered to be statistically significant.

\section{Discussion}

It is well known that tumor cell-derived VEGF is a key factor that acts on endothelial cells to promote angiogenesis, tumor growth and metastasis. Targeting proangiogenic mediators such as VEGF/VEGFR has emerged as a promising anticancer treatment strategy. In particular, VEGF fusion proteins have become an important aspect in novel cancer treatment strategies (20). In the present study, we constructed a protein containing VEGF $_{165}$ fused to melittin $\left(\mathrm{VEGF}_{165}\right.$-melittin). Successful expression of active $V_{E G F}{ }_{165}$-melittin was achieved in Pichia pastoris with yields $>80 \mathrm{mg} / \mathrm{l}$. N-terminal sequencing and mass spectrometric analysis verified that the fusion toxin was expressed and purified as expected. MTT and xenografts assays demonstrated that $\mathrm{VEGF}_{165}-$ melittin inhibited tumor growth in vivo and in vitro.

Among the identified proangiogenic regulators, VEGF, particularly VEGF-A and its two tyrosine kinase receptors, fms-like tyrosine kinase receptor (Flt1 and VEGFR-1) and kinase insert domain-containing receptor (KDR/FLK1 and VEGFR-2), have been identified as key mediators of the regulation of pathologic blood vessel growth and maintenance (21). In our results, $\mathrm{VEGF}_{165}$-melittin was more effective in HepG-2 than MHCC97-H cells. This diversity may be caused by differences in the VEGFR-1 and VEGFR-2 proteins expressed in HepG-2 and MHCC97-H cells. In subsequent studies, the expression of VEGF and the VEGF receptors (VEGFR-1 and VEGFR-2) was evaluated in HepG-2 and MHCC97-H cells by RT-PCR and western blot assays. Compared with the results we reported here, the $\mathrm{VEGF}_{165}$-melittin fusion toxin should be selective in targeting tumor cells that overexpress VEGFR-2. We hypothesize that the enhanced efficacy of VEGF fusion toxin may be due to the overexpression of VEGFR-2 in growing cells. Subsequently, 293 human primary embryonic kidney cells (293/KDR) overexpressing VEGFR-2 was constructed in our laboratory. VEGF $_{165}$-melittin inhibited growth of 293/ KDR cells at a dose of $6.4 \mu \mathrm{g} / \mathrm{ml}$. These effects were mediated by VEGFR-2, since the parental 293 cells lacking VEGFR-2 were not inhibited by fusion protein.

Melittin is a main component of bee venom. It is a small peptide with a linear structure composed of 26 amino acids (22). Bee venom has a wide range of effects including antibacterial, antiviral and anti-inflammatory effects; thus, it has been extensively used in the field of traditional medicine including treatments for back pain, rheumatism and skin diseases $(23,24)$. Furthermore, it has been shown that bee venom and/or melittin have inhibitory effects on the tumor growth of cervical, prostate, renal, breast, ovarian and liver tumor cells $(10,25,26)$. The results of our research are in accordance with previous studies demonstrating the suppression of melittin on the growth of human hepatic carcinoma cell lines $(11,27)$. Additionally, the fusion toxin $\mathrm{VEGF}_{165}$-melittin inhibited the proliferation of human hepatocellular carcinoma cell lines (HepG-2 and MHCC97-H) in a concentration-dependent manner. The most effective inhibitory concentration of $\mathrm{VEGF}_{165}$-melittin was $6.4 \mu \mathrm{g} / \mathrm{ml}$, resulting in an inhibition ratio of $52.3 \%$. The remarkable suppressive effects on cell proliferation were observed after $48 \mathrm{~h}$ in the experimental group. The present study indicated that the fusion toxin directly inhibits the growth of hepG-2 human hepatocellular carcinoma cells in vitro and in vivo. In follow-up experiments, more studies will be designed to detect the antitumor activity and mechanism of this fusion protein.

As a lower eukaryote, Pichia pastoris was identified as a suitable expression system for various recombinant proteins that retains biological activity with high quantity yields, and it also offers the benefits of $E$. coli (cost-effective and easy scale-up). In addition, the advantages of expression in a eukaryotic system include proper protein processing, folding and post-translational modifications $(28,29)$. In addition, Pichia pastoris does not secrete large amounts of intrinsic proteins, resulting in the easy isolation of foreign proteins. In the present study, $\mathrm{VEGF}_{165}$-melittin production was performed in a 2-liter fermentor, with yields $>80 \mathrm{mg} / \mathrm{l}$. The successful expression and purification of the recombinant fusion toxin VEGF $_{165}$-melittin and its activity in human hepatocellular carcinoma cells demonstrates that the fusion protein has the potential to be used as a novel cancer treatment strategy. This is the first report to describe the secretory expression of a human vascular endothelial growth factor fused to melittin in Pichia pastoris.

\section{Acknowledgements}

The present study was supported by grants from the Foundation for Distinguished Young Talents in Higher Education in Guangdong, China (LYM11080), the National Nature Science Foundation of China (No. 81101542) and the Guangdong Provincial Key Laboratory of Biotechnology Candidate Drug Research.

\section{References}

1. Carmeliet P and Jain RK: Angiogenesis in cancer and other diseases. Nature 407: 249-257, 2000.

2. Zhu W, Kato Y and Artemov D: Heterogeneity of tumor vasculature and antiangiogenic intervention: Insights from MR angiography and DCE-MRI. PLoS One 9: e86583, 2014.

3. Ferrara N and Ferrara N: Vascular endothelial growth factor: Molecular and biological aspects. Curr Top Microbiol Immunol 237: 1-30, 1999.

4. Hicklin DJ and Ellis LM: Role of the vascular endothelial growth factor pathway in tumor growth and angiogenesis. J Clin Oncol 23: 1011-1027, 2005.

5. Zhang D, Li B, Shi J, Zhao L, Zhang X, Wang C, Hou S, Qian W, Kou G, Wang H, et al: Suppression of tumor growth and metastasis by simultaneously blocking vascular endothelial growth factor (VEGF)-A and VEGF-C with a receptor-immunoglobulin fusion protein. Cancer Res 70: 2495-2503, 2010.

6. Chen AI and Advani RH: Beyond the guidelines in the treatment of peripheral T-cell lymphoma: New drug development. J Natl Compr Canc Netw 6: 428-435, 2008.

7. Tischer E, Mitchell R, Hartman T, Silva M, Gospodarowicz D, Fiddes JC and Abraham JA: The human gene for vascular endothelial growth factor. Multiple protein forms are encoded through alternative exon splicing. J Biol Chem 266: 11947-11954, 1991.

8. Houck KA, Leung DW, Rowland AM, Winer J and Ferrara N: Dual regulation of vascular endothelial growth factor bioavailability by genetic and proteolytic mechanisms. J Biol Chem 267 : 26031-26037, 1992. 
9. Koutsioumpa M, Poimenidi E, Pantazaka E, Theodoropoulou C, Skoura A, Megalooikonomou V, Kieffer N, Courty J, Mizumoto S, Sugahara K, et al: Receptor protein tyrosine phosphatase beta/ zeta is a functional binding partner for vascular endothelial growth factor. Mol Cancer 14: 19-34, 2015.

10. Oršolić N: Bee venom in cancer therapy. Cancer Metastasis Rev 31: 173-194, 2012.

11. Wang C, Chen T, Zhang N, Yang M, Li B, Lü X, Cao X and Ling C: Melittin, a major component of bee venom, sensitizes human hepatocellular carcinoma cells to tumor necrosis factorrelated apoptosis-inducing ligand (TRAIL)-induced apoptosis by activating CaMKII-TAK1-JNK/p38 and inhibiting I $\kappa \mathrm{B} \alpha$ kinaseNF-кB. J Biol Chem 284: 3804-3813, 2009.

12. Gajski G and Garaj-Vrhovac V: Melittin: A lytic peptide with anticancer properties. Environ Toxicol Pharmacol 36: 697-705, 2013.

13. Liu S, Yu M, He Y, Xiao L, Wang F, Song C, Sun S, Ling C and Xu Z: Melittin prevents liver cancer cell metastasis through inhibition of the Rac1-dependent pathway. Hepatology 47: 1964-1973, 2008.

14. Hoskin DW and Ramamoorthy A: Studies on anticancer activities of antimicrobial peptides. Biochim Biophys Acta 1778: 357-375, 2008.

15. Soman NR, Baldwin SL, Hu G, Marsh JN, Lanza GM, Heuser JE, Arbeit JM, Wickline SA and Schlesinger PH: Molecularly targeted nanocarriers deliver the cytolytic peptide melittin specifically to tumor cells in mice, reducing tumor growth. J Clin Invest 119: 2830-2842, 2009.

16. Lee MT, Chen FY and Huang HW: Molecular mechanism of Peptide-induced pores in membranes. Biochemistry 43 : 3590-3599, 2004.

17. Huh JE, Kang JW, Nam D, Baek YH, Choi DY, Park DS and Lee JD: Melittin suppresses VEGF-A-induced tumor growth by blocking VEGFR-2 and the COX-2-mediated MAPK signaling pathway. J Nat Prod 75: 1922-1929, 2012.

18. Shin JM, Jeong YJ, Cho HJ, Park KK, Chung IK, Lee IK, Kwak JY, Chang HW, Kim CH, Moon SK, et al: Melittin suppresses HIF-1 $\alpha /$ VEGF expression through inhibition of ERK and $\mathrm{mTOR} / \mathrm{p} 70 \mathrm{~S} 6 \mathrm{~K}$ pathway in human cervical carcinoma cells. PLoS One 8: e69380, 2013.

19. Pichia Expression Kit Version F: A Manual of Methods for Expression of Recombinant Proteins in Pichia pastoris. Invitrogen, 2002.
20. Ciomber A, Smagur A, Mitrus I, Cichoń T, Smolarczyk R, Sochanik A, Szala S and Jarosz M: Antitumor effects of recombinant antivascular protein ABRaA-VEGF121 combined with IL-12 gene therapy. Arch Immunol Ther Exp (Warsz) 62: 161-168, 2014.

21. Xu WW, Li B, Lam AK, Tsao SW, Law SY, Chan KW, Yuan QJ and Cheung AL: Targeting VEGFR1- and VEGFR2-expressing non-tumor cells is essential for esophageal cancer therapy. Oncotarget 6: 1790-1805, 2015.

22. Liu M, Zong J, Liu Z, Li L, Zheng X, Wang B and Sun G: A novel melittin-MhIL-2 fusion protein inhibits the growth of human ovarian cancer SKOV 3 cells in vitro and in vivo tumor growth. Cancer Immunol Immunother 62: 889-895, 2013.

23. Lee MT, Sun TL, Hung WC and Huang HW: Process of inducing pores in membranes by melittin. Proc Natl Acad Sci USA 110: 14243-14248, 2013.

24. Sommer A, Fries A, Cornelsen I, Speck N, Koch-Nolte F, Gimpl G, Andrä J, Bhakdi S and Reiss K: Melittin modulates keratinocyte function through $\mathrm{P} 2$ receptor-dependent ADAM activation. J Biol Chem 287: 23678-23689, 2012.

25. Qu L, Jiang M, Li Z, Pu F, Gong L, Sun L, Gong R, Ji G and Si J: Inhibitory effect of biosynthetic nanoscale peptide Melittin on hepatocellular carcinoma, driven by survivin promoter. J Biomed Nanotechnol 10: 695-706, 2014

26. Jo M, Park MH, Kollipara PS, An BJ, Song HS, Han SB, Kim JH, Song MJ and Hong JT: Anti-cancer effect of bee venom toxin and melittin in ovarian cancer cells through induction of death receptors and inhibition of JAK2/STAT3 pathway. Toxicol Appl Pharmacol 258: 72-81, 2012.

27. Liu H, Han Y, Fu H, Liu M, Wu J, Chen X, Zhang S and Chen Y: Construction and expression of sTRAIL-melittin combining enhanced anticancer activity with antibacterial activity in Escherichia coli. Appl Microbiol Biotechnol 97: 2877-2884, 2013.

28. Su M, Chang W, Cui M, Lin Y, Wu S and Xu T: Expression and anticancer activity analysis of recombinant human uPA1-43melittin. Int J Oncol 46: 619-626, 2015.

29. Wang DD, Su MM, Sun Y, Huang SL, Wang J and Yan WQ: Expression, purification and characterization of a human single-chain Fv antibody fragment fused with the Fc of an IgG1 targeting a rabies antigen in Pichia pastoris. Protein Expr Purif 86: 75-81, 2012. 